\title{
Lumbar Disc Herniation Associated with Contralateral Neurological Deficit: Can Venous Congestion Be the Cause?
}

\author{
Orhan Kalemci, Ceren Kizmazoglu, Ercan Ozer, Mehmet Nuri Arda \\ Department of Neurosurgery, Dokuz Eylül Üniversity School of Medicine, Inciralti, Izmir, Turkey
}

Lumbar disc herniation (LDH) associated with a contralateral neurological deficit is sometimes encountered by surgeons. Compression against the opposite pedicle in case of a large discal herniation and prominent stenotic changes of contralateral side are held responsible for contralateral symptoms and findings. In this study, we report a case of LDH associated with a painless contralateral neurological deficit. Prominent venous engorgement and congestion at the contralateral side of discal herniation were detected during the operation. It's treatment with bipolar coagulation and significant improvement was seen after the operation.

Keywords: Deficit; Neurologic; Venous congestion

\section{Introduction}

Lumbar disc herniation (LDH) causes radiculopathy of the same side. Midline discs are associated with bilateral symptomatology and findings. A LDH causing contralateral radiculopathy is sometimes encountered by surgeons [1,2]. In this study we report a left sided L4-5 disc herniation case, which caused radiculopathy and pain on the left side, but also painless drop foot on the right side. Prominent venous congestion was found during the operation. In this report, venous congestion as a cause of associated contralateral neurological deficit is discussed.

\section{Case Report}

A 74 year-old female patient was admitted to our outpatient clinic because of low back and left leg pain. She was suffering from $\mathrm{LDH}$ for approximately 4 years. Her complaints recurred during the last two weeks before admission to the outpatient clinic. Conservative treatment measures including physical therapy failed and she was consulted for surgery. Neurological examination revealed foot drop (2/5) on the right side and a positive straight leg rising test at 30 degrees on the left side. Magnetic resonance imaging (MRI) demonstrated L4-5 left paramedian discal herniation (Fig. 1). Neurological deficit of the patient was on the right side in contrast to a left sided herniation. Surgery was offered and the patient accepted the operation. At the operation, left sided hemilaminectomy, extruded fragment removal and discectomy were initially performed. Because of the right sided neurological deficit, exploration of the right side had been decided and at the operation it was seen that there was prominent venous engorgement and congestion in the epidural space and the right L5 nerve root was seen as it embedded. Venous structures were cauterized with bipolar forceps. No discal protrusion was detected on the right

Received Jan 7, 2011; Revised Aug 19, 2012; Accepted Aug 20, 2012

Corresponding author: Orhan Kalemci

Department of Neurosurgery, Dokuz Eylül Üniversitesi, Beyin ve Sinir Cerrahisi Anabilim Dalı, 35330 İnciralt1, İzmir, Turkey

Tel: +90-232-4123305, Fax: +90-232-2788802,E-mail: okalemci@gmail.com 
side. Following the surgery the patient's pain was resolved but some remained on the lower back due to incision and surgery. The right sided neurological deficit improved to $4 / 5$ in a few days. The patient was discharged after an uneventful postoperative period. The patient was completely free of pain and neurological deficit at the first year follow-up.

\section{Discussion}

LDH is generally associated with same side pain and neurological findings. Sometimes surgeons encounter a LDH causing contralateral radiculopathy associated with pain. Some cases having contralateral findings have been reported in the literature $[1,2]$. The cause of contralateral radiculopathy is attributed to prominent spondylotic changes and stenosis contralateral to the side of disc herniation associated with anatomical anomalies of lumbar nerve roots [2]. The disc herniation causes displacement and impaction of the dural sac with the emerging nerve roots in a narrowed lateral recess. In a normal spinal canal, compression against the opposite pedicle can cause contralateral leg pain [1]. In our case, there was a left sided paramedian disc herniation. Although facet joint changes were seen on MRI, the anterior-posterior diameter of the spinal canal was normal and epidural fat tissue can be seen radiologically. It was not large enough to cause compression against contralateral anatomical structures. After left sided disc excision, right sided hemilaminectomy and foraminotomy was also performed. Significant epidural venous plexus engorgement and congestion was detected on the right side during the operation. It was much more prominent than the general appearance of the epidural venous plexus. The possible obstruction of venous circulation by disc herniation caused venous congestion. This congestion might be the cause of the contralateral neurological deficit by causing compression in this case. The congested epidural venous plexus was cauterized with bipolar forceps. The patient's neurological deficit improved after surgery. It is not obvious whether its improvement came from disc removal or bipolar cauterization of the venous plexus. The postoperative cauda equina syndrome was attributed to venous congestion in a study by Henriques et al. [3]. They claimed venous congestion because they found no radiological abnormality regarding the cause of the cauda equina syndrome postoperatively in these cases. Venous congestion was also held responsible for a case of acute paraplegia developed after straining [4]. Also, in that case, there was intramedullary high signal intensity indicating compression. Since venous congestion is not detectable on MRI, no MRI abnormality regarding venous congestion was seen in our case. The mechanism as to how venous congestion causes a neurological deficit is obscure. First, it may be direct compression [4]. In an experimental study on porcine cauda equina, venous stasis was believed to impair nerve impulse propagation [5]. Because double level compression induced
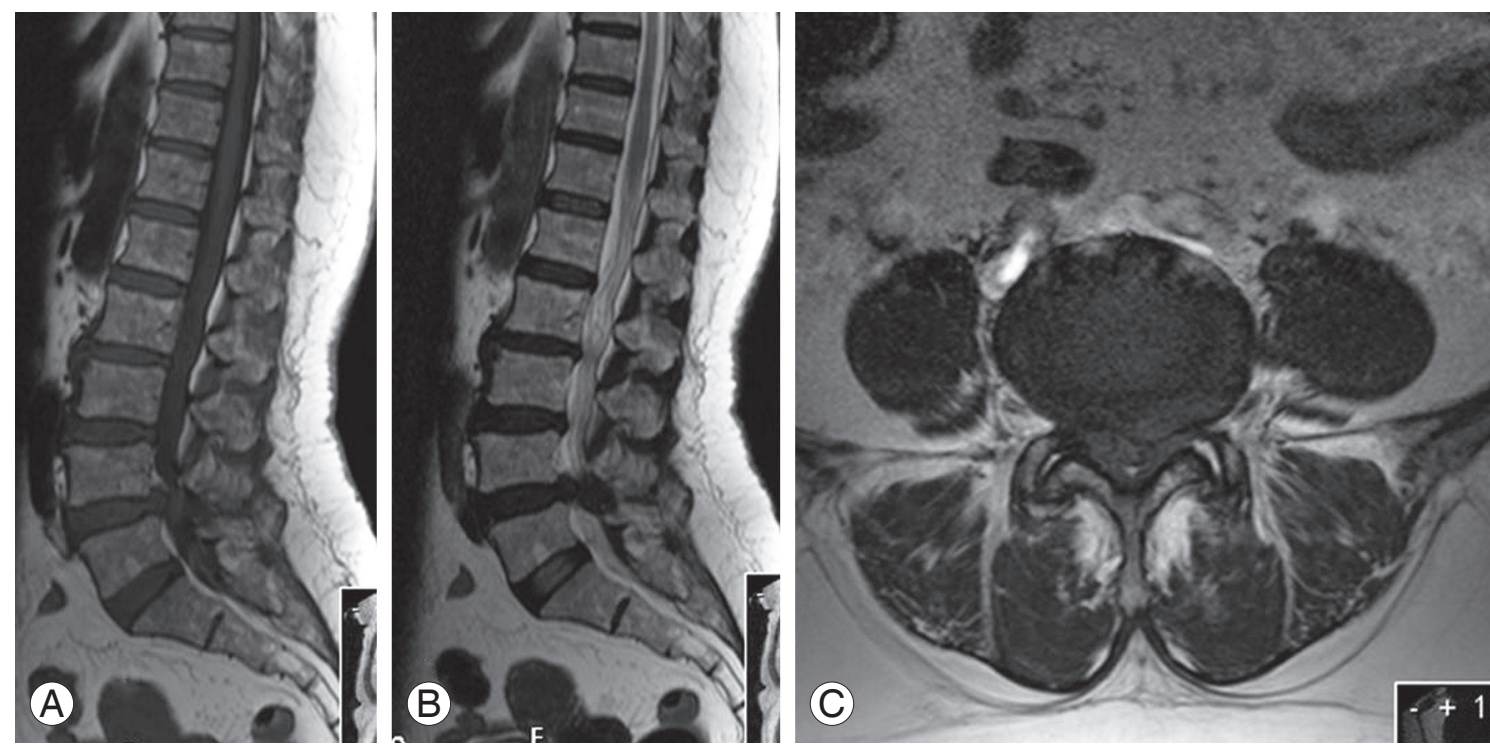

Fig. 1. Lumbar magnetic resonance imaging (MRI) of the patient. T1 and T2 weighed MRI scans (A, B) demonstrate L4-5 discal herniation. Axial T1 weighted image (C) shows left paramedian discal herniation. There is no finding attributable to venous congestion on these scans. 
a more pronounced effect on nerve than a single level. Nerve injury was further enhanced when the level of the compression sites were increased. Secondly, venous congestion can impair venous drainage and thus can cause venous stasis in the nerve tissue. A third mechanism may be neural fibrosis. Hoyland et al. [6] found a direct correlation between venous congestion and neural fibrosis in the intervertebral foramen. All these mechanisms may also take part in the nerulogical deficit process initiated by venous congestion.

A LDH sometimes causes a contralateral neurological deficit and venous congestion may be the cause. In the case of contralateral findings, hemilaminectomy and bipolar cauterization of the venous plexus may help in the improvement of neurological deficits.

\section{Conflict of interest}

No potential conflict of interest relevant to this article was reported.

\section{References}

1. Auld AW, DeWall JG. Myelographic defect on the side opposite the leg pain. A case report with an explanation of mechanism of action. Spine (Phila Pa 1976) 1979;4:174-5.

2. Choudhury AR, Taylor JC, Worthington BS, Whitaker R. Lumbar radiculopathy contralateral to upper lumbar disc herniation: report of 3 cases. Br J Surg 1978;65:8424.

3. Henriques T, Olerud C, Petren-Mallmin M, Ahl T. Cauda equina syndrome as a postoperative complication in five patients operated for lumbar disc herniation. Spine (Phila Pa 1976) 2001;26:293-7.

4. Yano S, Hida K, Seki T, Iwasaki Y, Akino M, Saitou H. A case of thoracic disc herniation with sudden onset paraplegia on toilet straining: case report. No Shinkei Geka 2003;31:1297-301.

5. Olmarker K, Rydevik B. Single- versus double-level nerve root compression. An experimental study on the porcine cauda equina with analyses of nerve impulse conduction properties. Clin Orthop Relat Res 1992;(279):35-9.

6. Hoyland JA, Freemont AJ, Jayson MI. Intervertebral foramen venous obstruction. A cause of periradicular fibrosis? Spine (Phila Pa 1976) 1989;14:558-68. 